\title{
Immunochemical Studies on Cell-wall Polysaccharide of Rough Mutants of Salmonella typhimurium
}

\author{
By A. A. LINDBERG \\ Department of Bacteriology, National Bacteriological Laboratory, Stockholm \\ AND T. HOLME \\ Department of Bacteriology, Karolinska Institutet, Stockholm \\ (Accepted for publication 17 November 1967 ) \\ SUMMARY \\ Immunological studies were made in order to characterize the lipopoly- \\ saccharides of rough mutants of Salmonella typhimurium and S. minnesota. \\ Groups of mutants, specified by their phage pattern, also showed group \\ homogeneity with respect to their immunological specificity, especially when \\ using the passive thaemagglutination inhibition technique. Cross-reactions \\ between mutants belonging to different phage groups could generally be \\ explained on the basis of the carbohydrate composition of the lipopoly- \\ saccharide.
}

\section{INTRODUCTION}

Lipopolysaccharides isolated from rough strains of different Salmonella species have been serologically differentiated by means of haemagglutination and haemagglutination inhibition techniques (Beckmann, Lüderitz \& Westphal, 1964; Lüderitz, Beckmann \& Westphal, I964; Lüderitz et al. I966a, b). In contrast to the numerous $\mathrm{O}$-specificities encountered in the wild-type O-antigens of different Salmonella strains only a few specificities are found in R-antigens regardless of the serotype from which the mutant has been derived. Rough mutants of Salmonella strains have also been defined by their sensitivity to rough-specific phages (Subbaiah \& Stocker, 1964; Wilkinson \& Stocker, 1964; Lüderitz et al. I966a; Brandis, I966). Wilkinson \& Stocker used a collection of eight R-specific phages, of which four were obtained from Brandis, in an attempt to separate rough mutants of Salmonella typhimurium strain LT 2. More than eight different phage patterns could be established. Results obtained by Lüderitz et al. (I966a) indicated a certain relationship between the phage pattern, the sugar composition of the lipopolysaccharide and the reactivity with R-antisera in groups of rough mutants from $S$. minnesota and $S$. ruiru. The present paper reports on the further characterization of the $S$. typhimurium rough mutants, investigated chemically in a preceding paper (Holme, Lindberg, Garegg \& Onn, I968), with the aid of serological techniques and the sensitivity to different phages.

\section{METHODS}

Strains. The Salmonella typhimurium and S. minnesota strains were the same as used in the preceding paper by Holme et al. (1968).

Lipopolysaccharides. The strains were grown and the lipopolysaccharides extracted 
from the bacteria by the phenol+water procedure (Westphal, Lüderitz \& Bister, I952). The lipopolysaccharides were purified by three cycles of washing and ultracentrifugation at $105,000 \mathrm{~g}$ for $4 \mathrm{hr}$ in a Christ Omega preparative ultracentrifuge. The pellet was lyophilized and the lipopolysaccharide resuspended in saline before use.

Antisera. Antisera were obtained by injecting rabbits intravenously three times a week for 9 weeks with heat-killed suspensions of the bacteria. Serum specific for the O-antigenic factor 4 of group B was obtained from the Salmonella Centre, National Bacteriological Laboratory, Stockholm. Serum against Salmonella typhimurium TV I I9, obtained by immunization of a horse, was obtained from Dr M. Raynaud (Institut Pasteur, Paris). All sera were inactivated at $56^{\circ}$ for $30 \mathrm{~min}$., absorbed with packed washed sheep red cells at $37^{\circ}$ for $2 \mathrm{hr}$ and stored at $-20^{\circ}$ until use.

Passive haemagglutination. Sheep red cells were collected aseptically in sterile Alsever solution and washed three times in phosphate buffered saline before use. The lipopolysaccharides were treated with sodium hydroxide to enhance the affinity to red cells. The procedure was done according to Beckmann et al. (I964).

For sensitization Io $\mathrm{ml}$. saline containing $250 \mu \mathrm{g}$. alkali-treated lipopolysaccharide were added to $\mathrm{I} \cdot 25 \mathrm{ml}$. of a $4 \%(\mathrm{v} / \mathrm{v})$ suspension of washed sheep red cells. After incubation at $37^{\circ}$ for $30 \mathrm{~min}$. the sensitized red cells were washed 3 times with saline and finally diluted to $10 \mathrm{ml}$. with phosphate-buffered saline. Serial twofold dilutions of serum were prepared with phosphate-buffered saline $(\mathrm{pH} 7)$. To each tube containing $0.2 \mathrm{ml}$. serum dilution an equal volume of sensitized red cells was added. The tubes were kept at room temperature and read after 3 and $18 \mathrm{hr}$. The haemagglutinating titre was determined as the last tube showing macroscopically visible haemagglutination. Serum with unsensitized sheep red cells, and sensitized red cells in saline only, were used as controls in each experiment. The reactions were identical or deviated in only one dilution step on repeated tests.

Haemagglutination inhibition. A twofold dilution series of the lipopolysaccharide used as inhibitor was added to appropriately diluted sera. The serum dilution used was 4-8 haemagglutinating units (serum diluted 4-8 times less than the highest dilution causing haemagglutination). Serial twofold dilutions of the lipopolysaccharide with concentrations ranging from 256 to I $\mu \mathrm{g}$. $/ \mathrm{ml}$. were prepared in saline; $0.2 \mathrm{ml}$. of each lipopolysaccharide dilution was added to an equal volume of the appropriate serum dilution. The mixtures were incubated at $37^{\circ}$ in a water bath for $30 \mathrm{~min}$. Thereafter $0.2 \mathrm{ml}$. of sensitized sheep red cells was added to each mixture. Haemagglutination was read after incubation for $30 \mathrm{~min}$. at $37^{\circ}$ and $3 \mathrm{hr}$ at room temperature. The lowest concentration of lipopolysaccharide giving a total inhibition of the haemagglutination was determined. The haemagglutination titre without inhibition was determined in each experiment. Homologous inhibition with the lipopolysaccharides used for sensitization was also done, as well as the controls described in the preceding section.

Phage classification. The strains were characterized by their pattern of sensitivity to a collection of phages. The phages used were classified as smooth-specific (P 22), rough specific ( $\mathrm{P} 22 \mathrm{I}, 6 \mathrm{SR}, \mathrm{Br} 2, \mathrm{Ffm}, \mathrm{Br} 60$ and $\mathrm{C} 2 \mathrm{I}$ ) and phages active on smooth and rough strains (P $22 \mathrm{~h}$ and FO-I). The phages were obtained from Professor $\mathrm{H}$. Brandis (Hygiene-Institut der Universität Göttingen) and Professor B. A. D. Stocker (Dept. of Medical Microbiology, Stanford, California, U.S.A.). The phages were propagated by the soft-agar layer method of Adams (I959) or in broth culture. The 
lysates of the phages contained $10^{8}-10^{10}$ plaque-forming units (p.f.u.)/ml. Freshly prepared lawns of the strains on nutrient agar (Difco) were tested for sensitivity to tenfold dilution steps of the phages by the spot technique. Readings were carried out after 5 and $18 \mathrm{hr}$ incubation at $37^{\circ}$.

\section{RESULTS}

Phage classification of the strains

Drs Wilkinson \& Stocker (personal communication) used a collection of phages for grouping rough mutants of Salmonella typhimurium. Differences in the sensitivity

Table I. Phage patterns of (I) wild-type Salmonella typhimurium 395MS and derived mutants, (2) known mutants of S. typhimurium LT 2 and (3) known mutants of S. minnesota s99. Phages: smooth specific ( $P$ 22), smooth and rough specific $(P 22 h, F O-I)$ and rough specific ( $P$ 22I, $6 \mathrm{SR}, \mathrm{Br} 2, \mathrm{Ffm}, \mathrm{Br} 60, \mathrm{C} \mathrm{2I})$.

Phage patterns obtained by using the collection of nine phages in spot test on the $S$. typhimurium and $S$. minnesota strains. +, Clear or turbid confluent lysis with the phage undiluted, scattered plaques in higher dilutions. -, No lytic effect of the phages.

\begin{tabular}{|c|c|c|c|c|c|c|c|c|c|c|}
\hline \multirow[b]{2}{*}{$\begin{array}{c}\text { Strain } \\
\text { S.typhimurium }\end{array}$} & \multicolumn{9}{|c|}{ Phage } & \multirow[b]{2}{*}{ Classification } \\
\hline & \multicolumn{3}{|c|}{$\mathrm{P}_{22} \mathrm{P}_{22} \mathrm{~h}$ FO-I } & \multicolumn{2}{|c|}{ P22I $6 \mathrm{SR}$} & Br 2 & \multicolumn{2}{|c|}{ Ffm Br 60} & $\mathrm{C}_{2 \mathrm{I}}$ & \\
\hline $395 \mathrm{MS}$ & + & + & + & - & - & - & - & - & - & Smooth \\
\hline 395 MRO & - & - & + & + & + & + & + & + & - & Rough sensitive \\
\hline 395 MR I & - & - & - & + & - & + & + & + & - & Rough resistant-I \\
\hline $395 \mathrm{MR} 2$ & - & - & + & + & + & + & + & + & - & Rough sensitive \\
\hline $395 \mathrm{MR} 3$ & - & - & + & + & + & + & + & + & - & Rough sensitive \\
\hline 395 MR 4 & - & - & + & + & + & + & + & + & - & Rough sensitive \\
\hline 395 MR 5 & - & - & - & + & - & - & + & + & - & Rough resistant-2 \\
\hline 395 MR 6 & - & - & - & + & - & - & + & + & - & Rough resistant-2 \\
\hline $395 \mathrm{MR} 7$ & - & - & - & - & - & - & + & + & - & Rough resistant-2 \\
\hline $395 \mathrm{MR} 8$ & - & - & - & + & - & - & + & + & - & Rough resistant-2 \\
\hline $395 \mathrm{MR} 9$ & - & - & \pm & + & - & - & + & + & + & Galactose-epimerase-less \\
\hline 395 MR IO & - & - & - & + & - & - & + & + & + & Glucosyl-I-transferase-less \\
\hline TV I 19 & - & - & + & + & + & + & + & + & - & Rough sensitive \\
\hline TV I 48 & - & - & - & + & - & - & + & + & - & Rough resistant-2 \\
\hline TV 160 & - & - & - & + & - & + & + & + & - & Rough resistant-I \\
\hline TV 161 & - & - & - & + & - & + & + & + & - & Rough resistant-I \\
\hline TV I63 & - & - & + & + & + & + & + & + & - & Rough sensitive \\
\hline TVI66 & - & 一 & - & + & - & + & + & + & - & Rough resistant-I \\
\hline TV 208 & + & + & + & + & + & - & - & + & - & Not recognized \\
\hline TV 225 & - & - & + & + & + & + & + & + & - & Rough sensitive \\
\hline TV 226 & - & - & + & + & + & + & + & + & - & Rough sensitive \\
\hline TV 227 & - & - & + & + & + & + & + & + & - & Rough sensitive \\
\hline \multicolumn{11}{|l|}{ S. minnesota } \\
\hline s99 & - & - & + & - & - & - & - & - & - & Not recognized \\
\hline$R 60$ & - & - & + & - & - & + & + & + & - & Rough sensitive \\
\hline R 345 & - & - & - & - & - & + & + & + & - & Rough resistant-I \\
\hline
\end{tabular}

to the various phages may depend on the composition of the cell-wall lipopolysaccharide of the mutants. Thus, the sensitivity to phage P 22 was limited to the smooth strains of Salmonella having the O-antigen I2 (Zinder \& Lederberg, 1952). Phage FO-I lysed smooth strains and mutants belonging to chemotype $\mathrm{Ra}$ and was therefore designated as a SR-specific phage. Six phages lysed only rough mutants and were 
named R-specific. The strains used in the present work were grouped on the basis of their sensitivity pattern to nine phages. The phage patterns are represented in Table $\mathrm{I}$. The designation of the patterns is in accordance with Wilkinson \& Stocker (1964).

Smooth specific. The parent strain, Salmonella typhimurium 395MS was lysed by the smooth specific phage $\mathrm{P} 22$ and the smooth and rough specific phages $\mathrm{P} 22 \mathrm{~h}$ and FO-I. The phage pattern of strain TV 208 was not recognized since the strain was lysed by phages from all three groups.

Rough sensitive. Nine of the ten strains included in this group displayed a pattern characterized by lysis with the phages FO-I, $\mathrm{P} 22 \mathrm{I}, 6 \mathrm{SR}, \mathrm{Br} 2, \mathrm{Ffm}$ and $\mathrm{Br} 60$. Salmonella minnesota $\mathrm{R} 60$ was lysed only by the phages $\mathrm{FO}-\mathrm{I}, \mathrm{Br} 2, \mathrm{Ffm}$ and $\mathrm{Br} 60$, but adsorbed the phages $\mathrm{P} 22 \mathrm{I}$ and $6 \mathrm{SR}$. Therefore this strain was classified as rough sensitive.

Rough resistant-I. Mutants in this group were lysed by the rough specific phages P 22I, Br 2, Ffm and Br 60. This group contained four strains: RI, TV I60, TVI6I and TV I66. Salmonella minnesota R 345 was included in this group since it adsorbed phage $\mathbf{P} 22 \mathrm{I}$.

Rough resistant-2. The four strains in this group, $\mathrm{R} 5, \mathrm{R} 6, \mathrm{R} 8$ and $\mathrm{TV} \mathrm{I} 48$, were separated from the rough resistant-I group by their resistance to phage $\mathrm{Br} 2$. The mutant 395MR 7 was included in this phage pattern although it was resistant to phage P 22 I since the receptor structure for this phage appears to be independent of the polysaccharide structure (unpublished).

UDP-Galactose-4-epimerase-less mutant pattern. Only one strain, R9, was represented in this class. This galactose non-fermenter was sensitive to phages $\mathrm{P} 22 \mathrm{I}$ Ffm, $\mathrm{Br} 60$ and $\mathrm{C} 2 \mathrm{I}$.

Glucosyl-I-transferase-less mutant pattern. The mutant $\mathrm{R}$ IO showed the same phage pattern as strain R9. The designation glucosyl-I-transferase-less is based on the phage pattern, its ability to ferment galactose and the presence of S-specific polysaccharides in the cytoplasmic fraction.

\section{Haemagglutination and haemagglutination inhibition reactions}

The lipopolysaccharides of the Salmonella typhimurium and S. minnesota strains were tested against II antisera with passive haemagglutination technique (Table 2). The homologous haemagglutination titres ranged between I/640 and I/ I280. Duplicate or triplicate determinations were made in all experiments with positive reactions. The variability did not in any case exceed one dilution step. Sera drawn from rabbits before immunization showed no reactions in dilution exceeding $1 / 40$. Titres $1 / 160$ or above were considered as significantly positive reactions.

The lipopolysaccharides were further investigated in haemagglutination inhibition tests against eight homologous systems, representative for the smooth form and the different groups of rough mutants. Homologous inhibition was observed with the lipopolysaccharides in concentrations ranging from less than I to $4 \mu \mathrm{g}$. $/ \mathrm{ml}$. (Table 3 ).

The results of the immunological studies will be presented in a following section of this paper. 


\section{Table 2. Passive haemagglutination of Salmonella antisera with Salmonella lipopolysaccharides}

Haemagglutination systems. Data recorded are the highest dilution of serum, before addition of the lipopolysaccharide sensitized erythrocytes, giving visible haemagglutination. Entries left blank indicate negative reaction or reaction only in the dilution $1 / 40$.

\begin{tabular}{|c|c|c|c|c|c|c|c|c|c|}
\hline \multirow[b]{2}{*}{ Lipopolysaccharide } & \multirow{2}{*}{$\begin{array}{c}\text { Salmonella } \\
\text { factor } \\
0-4\end{array}$} & \multicolumn{6}{|c|}{$\begin{array}{c}\text { Reciprocal of titre of antiserum to } \\
\text { S. typhimurium }\end{array}$} & \multicolumn{2}{|c|}{ S. minnesota } \\
\hline & & TV 208 & TVII9 & TVI 63 & TV I 60 & R6 & R9 & $\mathrm{R} 60$ & R 345 \\
\hline \multicolumn{10}{|l|}{ S.typhimurium } \\
\hline $395 \mathrm{MS}$ & 1280 & 160 & 320 & 80 & 80 & . & 160 & . & . \\
\hline 395 MRO & . & & I 280 & 640 & & & & 80 & . \\
\hline 395 MR I & . & 80 & • & $\therefore$ & 160 & 160 & 80 & . & 80 \\
\hline 395 MR 2 & . & . & 1280 & 640 & . & . & . & 160 & . \\
\hline 395 MR 3 & . & 80 & 1280 & 640 & . & 80 & . & 320 & . \\
\hline 395 MR 4 & 160 & . & 1280 & 640 & . & . & . & 160 & . \\
\hline 395 MR 5 & 160 & 80 & 80 & . & 160 & 160 & 160 & . & . \\
\hline 395 MR 6 & . & . & . & . & . & 640 & . & . & . \\
\hline 395 MR 7 & . & . & . & . & . & 320 & . & . & . \\
\hline 395 MR 8 & . & . & . & . & . & 160 & . & . & - \\
\hline 395 MR9 & 320 & . & . & . & 80 & 320 & 640 & . & . \\
\hline 395 MR IO & . & . & . & . & . & . & . & . & . \\
\hline TV 160 & . & . & . & . & 640 & 80 & . & . & 80 \\
\hline TV163 & 320 & 80 & I 280 & 1280 & 160 & 80 & 80 & . & . \\
\hline TV 208 & 640 & 640 & 160 & - & 160 & 80 & 160 & 80 & . \\
\hline \multicolumn{10}{|l|}{ S. minnesota } \\
\hline $\mathrm{R} 60$ & . & . & 1280 & I 280 & 80 & 80 & . & 640 & . \\
\hline R345 & . & . & . & . & 160 & 80 & . & . & 640 \\
\hline
\end{tabular}

Table 3. Haemagglutination inhibition of Salmonella antisera with Salmonella lipopolysaccharides

Haemagglutination inhibition systems : homologous lipopolysaccharide and serum. Data recorded are the minimal concentration $(\mu \mathrm{g} . / \mathrm{ml}$.) of lipopolysaccharide from the $S$. typhimurium and $S$. minnesota strains needed for inhibition of the homologous system. Entries left blank indicate that lipopolysaccharide concentrations higher than $256 \mu \mathrm{g} . / \mathrm{ml}$. were needed for the inhibition.

\begin{tabular}{|c|c|c|c|c|c|c|c|}
\hline \multirow[b]{2}{*}{ Lipopolysaccharide } & \multicolumn{5}{|c|}{ S. typhimurium } & \multicolumn{2}{|c|}{ S. minnesota } \\
\hline & TV 208 & TV I63 & TV 160 & $\mathrm{R} 6$ & R9 & R 60 & R345 \\
\hline \multicolumn{8}{|l|}{ S.typhimurium } \\
\hline $395 \mathrm{MS}$ & I6 & . & . & . & . & . & . \\
\hline 395 MRO & . & 8 & . & . & . & . & . \\
\hline 395 MR I & . & . & 8 & . & . & . & . \\
\hline 395 MR 2 & . & 8 & . & . & . & . & . \\
\hline 395 MR 3 & . & 4 & . & . & . & - & . \\
\hline $395 \mathrm{MR} 4$ & . & 8 & . & . & . & . & . \\
\hline 395 MR 5 & . & . & . & 4 & . & . & . \\
\hline 395 MR 6 & . & . & . & $<\mathrm{I}$ & 64 & . & . \\
\hline $395 \mathrm{MR} 7$ & . & . & . & 32 &. & . & . \\
\hline $395 \mathrm{MR} 8$ & . & . & . & 4 & 32 & . & . \\
\hline 395 MR 9 & . & . & . & . & $<\mathrm{I}$ & . & . \\
\hline 395 MR IO & . & . & . & . & 128 & . & . \\
\hline TVI 60 & . & . & 4 & . & . & . & . \\
\hline TV I 63 & . & 4 & . & . & . & . &. \\
\hline TV 208 & 4 & . & . & . & . & $\dot{.}$ & . \\
\hline \multicolumn{8}{|l|}{ S. minnesota } \\
\hline R 60 & . & 8 & . & . & . & $<\mathrm{I}$ & . \\
\hline R 345 & . & - & . & . & 128 & . & $<\mathbf{I}$ \\
\hline
\end{tabular}




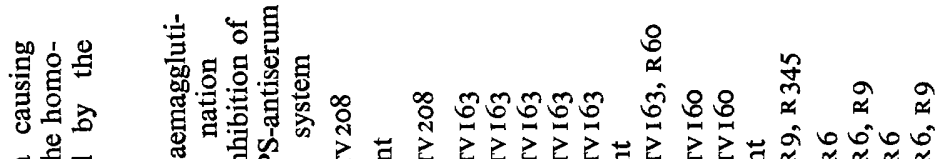

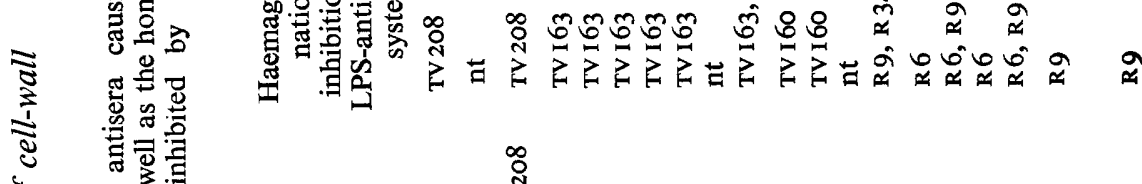

bे

:

문

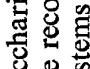

苋管

¿을

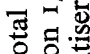

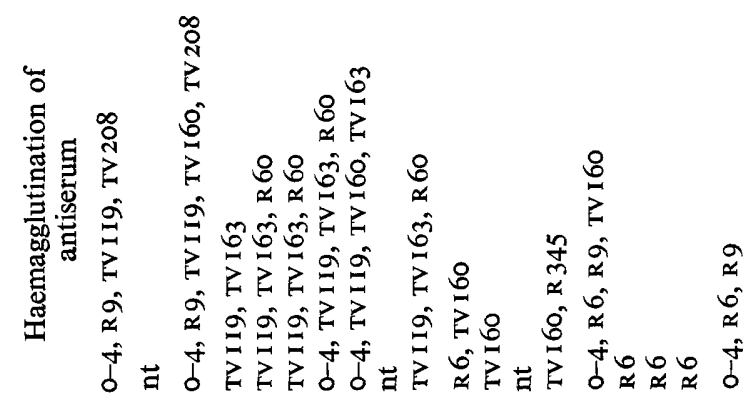

$\therefore$ क.

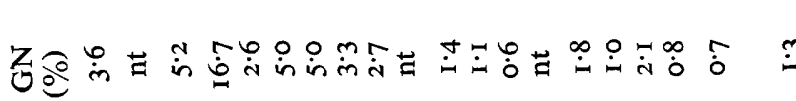

过.

구을

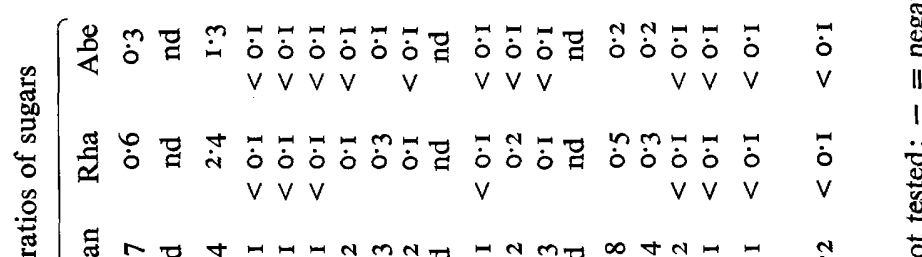

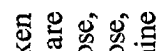

西

0

尊 il

究

ฐ

造

蛋

তే

寻-



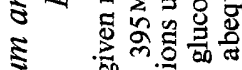

.



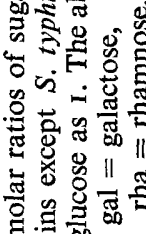

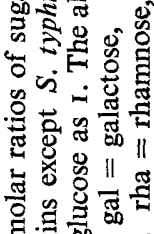

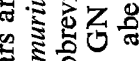

वं

菊

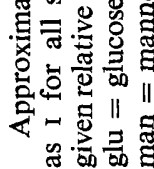

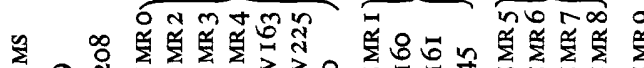

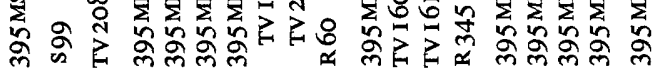
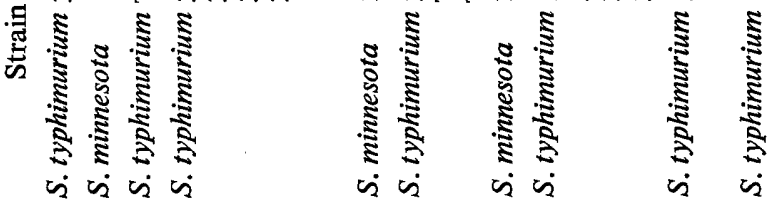


\section{Correlation between chemical composition, sensitivity to phages and immunochemical investigations}

In Table 4 the results of the different methods used for characterization of the rough mutants are summarized. The phage patterns which were obtained have been used as a basis for classification because they offered the most simple overall view.

Smooth specific pattern. The cell-wall polysaccharide from the parent strain Salmonella typhimurium 395 MS contained small amounts of ribose and the S-specific sugars. The phage pattern was smooth specific. In haemagglutination tests positive reactions were observed with sera against the O-antigen 4, the Ra mutants TV II9 and TV 208 as well as the Rc mutant R9. Haemagglutination inhibition showed a cross-reaction with the TV 208 system. Salmonella minnesota $S 99$, only lysed by the FO-I phage, had heptose:glucose: galactose ratios of $I: I: 2 \cdot 4$.

Salmonella typhimurium TV 208. This strain was originally described as a Ra mutant (Nikaido, Nikaido, Subbaiah \& Stocker, I964). The lipopolysaccharide contained great amounts of glucose, galactose, mannose, rhamnose and abequose compared to the other Ra-mutants. In serological tests TV 208 showed cross-reactions with $\mathrm{S}$-specific as well as several R-specific sera. Also in phage typing the strain behaved as both a smooth and rough strain. Its sensitivity to the phage $\mathrm{P} 22$ indicated the presence of antigen 12 of the S-specific repeating unit. In contrast to the smooth form the strain was sensitive to three of the rough-specific phages.

Rough sensitive pattern. Four of the mutants in this group, Ro, R 4, TV I63 and TV 225, had heptose:glucose:galactose molar ratios of $\mathrm{I}: \mathrm{I}: \mathrm{I}$ whereas for three of the mutants the figures varied from $I: 0 \cdot 5: 0 \cdot 5$ for $R 3, I: 0 \cdot 6: I$ for $R 2$ and $I: I \cdot 4: I \cdot 5$ for Salmonella minnesota $\mathrm{R} 60$. The content of $N$-acetyl-glucosamine in the Ro mutant was much higher than in any of the other $S$. typhimurium strains. Small amounts of mannose, rhamnose and abequose were found in all the mutants with the exception of $S$. minnesota $\mathrm{R} 60$. In serological tests the strains were fairly regular with one notable exception. The $S$. minnesota $\mathrm{R} 60$ homologous system was not inhibited by any of the lipopolysaccharides from the six $S$. typhimurium mutants of this group.

Rough resistant-I pattern. This group contained four strains, Salmonella typhimurium 395MRI, TVI60, TVI6I and $S$. minnesota R345. The polysaccharide composition differed from that of the rough sensitive group as the strains contained a significantly smaller amount of glucosamine. Again the S-specific sugars were detected in small amounts. In serological tests cross-reactions were detected with the TV I60 antiserum. Antiserum against $S$. minnesota R345, the test strain for RI specificity according to Lüderitz et al. (I966a), showed weak reactions in the haemagglutination test with the RI and TV I6o lipopolysaccharides.

Rough resistant-2 pattern. The phage pattern of strains in this group differed from the rough resistant-I group as the strains were not lysed by phage $\mathrm{Br} 2$. Salmonella typhimurium 395 MR 5 and R6, selected for their resistance to phage FO-I, had heptose: glucose:galactose molar ratios of approximately $\mathrm{r}: 0 \cdot 75: \mathrm{I}$ and appreciable amounts of mannose, rhamnose and abequose. In haemagglutination tests lipopolysaccharide from R 5 cross-reacted with O-factor 4, R6, R 9 and TV I 60 sera whereas R 6 only showed the homologous reaction. In haemagglutination inhibition tests lipopolysaccharide from $\mathrm{R} 6$ inhibited the $\mathrm{R} 9$ system. The mutants $\mathrm{R} 7$ and $\mathrm{R} 8$, selected for their resistance to the Lilleengen phage 4 , had heptose:glucose:galactose molar ratios of approxi- 
mately $I: 0.5: 0.5$ with smaller amounts of the S-specific sugars than the strains $R 5$ and $\mathrm{R} 6$. Lipopolysaccharide from $\mathrm{R} 7$ and $\mathrm{R} 8$ cross-reacted with $\mathrm{R} 6$ antisera in haemagglutination tests. The homologous systems $\mathrm{R} 6$ and $\mathrm{R} 9$ were both inhibited by lipopolysaccharide from $\mathrm{R} 8$.

UDP-Galactose-4-epimerase-less pattern. The only strain in this class, $\mathrm{R} 9$, was characterized by its sensitivity to phage $\mathrm{C}_{2} \mathrm{I}$ in addition to the phages $\mathrm{P}_{22 \mathrm{I}}, \mathrm{Ffm}$ and $\mathrm{Br} 60$. The polysaccharide of this strain contained heptose and glucose in a $\mathrm{I}: \mathrm{I}$ ratio and small amounts of galactose and S-specific sugars. Upon addition of galactose the strain $\mathrm{R} 9$ developed smooth specific characteristics as judged by colony morphology, agglutination reactions and phage pattern. Sometimes this strain showed an unexpected sensitivity to phage FO-I in galactose-free media but not to the phages $6 \mathrm{SR}$ and $\mathrm{Br} 2$ at the same time. In haemagglutination tests the lipopolysaccharide cross-reacted with O-factor 4 and $\mathrm{R} 6$ antisera.

Glucosyl-I-transferase-less mutant pattern. Salmonella typhimurium 395MR Io was a galactose-fermenter with the same phage pattern as the UDP-galactose-epimeraseless mutant R9. Its polysaccharide contained small amounts of galactose, mannose and traces of glucose, rhamnose and abequose. The $R I O$ lipopolysaccharide did not show cross-reactions in haemagglutination tests but inhibited the $\mathrm{R} 9$ system in high concentrations.

\section{DISCUSSION}

Attempts to classify rough mutants of Salmonella according to both the chemical composition, serological specificity and sensitivity to phages were made on Salmonella minnesota and S. ruiru mutants by Lüderitz et al. (1966a). The sugar composition of the lipopolysaccharides of the rough mutants separated them into five groups. Haemagglutination inhibition tests revealed that many antisera cross-reacted with lipopolysaccharides extracted from rough mutants belonging to different chemotypes.

The results presented in a preceding paper (Holme et al. 1968) showed that the rough mutants of Salmonella typhimurium in addition to the sugars of the basal core also contained the S-specific sugars in varying amounts. It was not possible, on the basis of the molar ratios of the sugar components calculated for the different S. typhimurium and $S$. minnesota rough mutants, to separate the strains into distinct groups. In haemagglutination and haemagglutination inhibition tests, presented in this paper, the lipopolysaccharides of the different rough strains showed a variety of cross-reactions which also made a separation between the mutants difficult. The overall picture was therefore based on the phage patterns obtained when applying the collection of phages used in an investigation of the phage susceptibility of mutants of $S$. typhimurium LT 2 by Drs Wilkinson \& Stocker (personal communication).

With the use of this collection of nine phages the rough mutants of Salmonella typhimurium were separated into five groups. Two of the $S$. typhimurium mutants had phage patterns which had not been recognized earlier. The three $S$. minnesota strains also showed phage patterns not recognized earlier but were included into the groups as they adsorbed the corresponding phages. Each phage pattern was stable when the strains were retyped several times as well as when the strains were grown both in the absence and in the presence of glucose. This indicated that the mutants were stable rough forms as revertants to the smooth form would have given growth in the area lysed by the R-specific phages in spot tests. 
The mutant Salmonella typhimurium TV 208 was lysed by both the S-specific and some of the R-specific phages. This strain was originally described as a $\mathrm{Ra}$ mutant defective in the synthesis of TDP-rhamnose (Nikaido et al. 1964). These workers also suggested that strain TV 208 showed a high frequency of reversion to the smooth form. The molar ratios of the sugar components of the TV 208 polysaccharide revealed high amounts of the S-specific sugars as compared to the other Ra mutants. In serological investigations the strain showed both $R$ and $S$ specificity. The results would be compatible with the assumption that the observed S-specificity could be due to smooth revertants. In virulence tests, however, no mice were killed by strain TV 208 ; if smooth revertants were frequent in cultures of $\mathrm{T}, \mathrm{V}, 208$ no mice would have survived. It appears that the strain TV 208 is a non-virulent rough mutant with unusually high content of S-specific sugars, which confer susceptibility to the S-specific phage P 22 and serological cross-reactions with the smooth form. In the rough sensitive group the sugar composition was similar for four of the strains-Ro, R 4, TVI63, TV 225-with heptose:glucose:galactose ratios of I : I : I, more than $2.5 \%$ of glucosamine in the polysaccharide and small amounts of mannose, rhamnose and abequose. Two of the mutants-R2 and R3-did not show the I:I:I ratios. The observed differences in carbohydrate composition were, however, not reflected in the serological reactions where the strains were quite homogeneous with the exception of Salmonella minnesota $\mathrm{R} 60$.

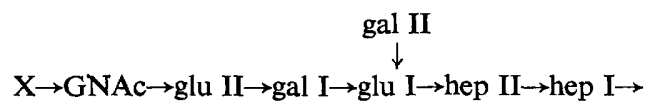

Fig. I. Possible structure of core of Salmonella lipopolysaccharide. Hep $=$ heptose, glu $=$ glucose, gal $=$ galactose, GNAc $=N$-acetyl glucosamine, $\mathrm{X}=$ unidentified unit, possibly involved in RII specificity.

The lipopolysaccharides of all the Ra mutants inhibited the homologous S. typhimurium TV 163 system in haemagglutination inhibition tests whereas the homologous $S$. minnesota R60 system was not inhibited by any of the $S$. typhimurium lipopolysaccharides. This non-reciprocal inhibition could be attributed to the possible attachment of an acid-labile unit ' $\mathrm{X}$ ' to the terminal $\mathrm{N}$-acetylglucosamine of the core polysaccharide (Fig. I). This unit is supposed to be involved in the production of the complete core by $r f b$ mutants of the antigenic R II character, i.e. S. minnesota R60 (Luideritz et al. I966a). The rfa mutant $S$. typhimurium TVI63 contains both the glucosamine of the core and the cytoplasmic S-specific polysaccharide. The latter has been demonstrated by gel precipitation (unpublished). This strain therefore should not possess the ' $X$ '-unit, which is indicated by the haemagglutination inhibition tests. However, as evidenced by the uniformity in phage pattern within this group the presence of the ' $\mathrm{X}$ '-unit does not appear to have any influence on the susceptibility of the strains to the different phages.

The eight mutants classified as chemotype $\mathrm{Rb}$ were complex in phage pattern, carbohydrate composition and serological specificity. The Salmonella typhimurium mutants with the rough resistant-I pattern had heptose:glucose:galactose ratios of approximately $\mathrm{I}: 0.5: \mathrm{I}$, which indicates that the core ended with the gal I-residue (Fig. I). The $S$. minnesota $\mathrm{R} 345$ mutant did not show the same sugar ratios and differed also in serological specificity. The mutants 395MR 5, R6, R7 and R 8 showed the rough resistant-2 pattern and were rather homogeneous in haemagglutination inhibition 
tests. The heptose:glucose:galactose ratios for the $\mathrm{R} 7$ and $\mathrm{R} 8$ mutants were approximately $1: 0 \cdot 5: 0.5$, which could be due to that either the gal I or gal II residue of the core is lacking. This would explain the difference between the two classes within the chemotype $\mathrm{Rb}$ with respect to phage pattern and serological specificity. The heptose: glucose:galactose ratios for the R5 and $\mathrm{R} 6$ mutants were not the same as for the R 7 and $R 8$ mutants, which could be due to a high degree of leakiness resulting in the synthesis of a few S-specific side chains. The side chains do not, however, prevent the reaction with $\mathrm{R}$-specific antisera.

The UDP-galactose-4-epimerase-less mutant R9 contained small amounts of galactose and S-specific sugars. Possible explanations of this finding were discussed in the preceding paper by Holme et al. (1968). The assumption that rhamnose, mannose and abequose were linked in a configuration determining $\mathrm{O}$-specificity was supported by the finding of cross-reactions between the anti R9 serum and lipopolysaccharides from the smooth specific strain Salmonella typhimurium 395MS and S. typhimurium TV 208. The lipopolysaccharide from R9 also reacted with the O-factor 4 serum in haemagglutination tests. Furthermore this strain showed an unexpected sensitivity to the FO-I phage on some occasions. This FO-I sensitivity was regularly accompanied by a lysis with phage $\mathrm{C} 2 \mathrm{I}$ which otherwise has been reported to lyse only mutants of the Rc and Rd chemotypes (Shedlovsky \& Brenner, I963; Stocker, Wilkinson \& Mäkelä, 1966). No lysis with phage $P 22$ or $P 22 \mathrm{~h}$ was ever observed. The fact that this strain could be sensitive to both FO-I and $\mathrm{C} 2 \mathrm{I}$ indicates that the FO-I receptostructure can be present without conferring a masking effect on the $\mathrm{C} 2 \mathrm{I}$ receptor structure. The same inconstancy in phage pattern has also been observed for the UDP-galactose-4-epimerase-less mutant $S$. typhimurium SL I070. Thus for the $S$. typhimurium mutants the phage pattern was constantly rough, the only exception being the strain TV208. The presence of small amounts of the S-specific sugars was not accompanied by any adsorption of phages $\mathbf{P} 22$ or $\mathbf{P} 22 \mathrm{~h}$.

Naide et al. (1965) described a class of mutants in Salmonella intermediate between the smooth and the rough form both in biological properties and lipopolysaccharide composition. They were named semi-rough (SR) mutants and displayed a smooth colony type on solid media, the O-antigen 4 was present in all mutants and their recombinants, and the $\mathrm{O}$-antigen 5 was detected in some. The S-specific sugars were present in small amounts in all the strains. Some of the strains used in the present investigation have characteristics which could indicate that they were SR-mutants, namely R 4, R 5 and TV I63. SR mutants display typical phage patterns in addition to the characteristics described above (Drs Wilkinson \& Stocker, personal communication). Both the mutants R 4 and TV 163 showed a phage pattern which was recognized as specific for the rough sensitive group. The mutants R 5 and TV I 63 synthesize the soluble hapten which was not found in any of the SR-mutants (Naide et al. 1965). The results indicate that none of the mutants could be classified as semi-rough using the definition of Naide et al. (1965).

In haemagglutination tests cross-reactions were observed between mutants belonging to different phage groups. The presence of the cross-reacting antigens was reflected in the monosaccharide composition of the lipopolysaccharide. With the haemagglutination inhibition technique the reactions were more specific for each phage group.

Additional information on the structure of the polysaccharide core may be obtained 
by the use of the different R-specific phages in inhibition experiments with lipopolysaccharides extracted from the rough mutants. The changing susceptibility to the rough specific phages observed for the different mutants indicates that the monosaccharide components determine the specificity of the phage receptors.

We are grateful to colleagues mentioned above for the generous supply of bacteriophages and sera, and to Dr R. G. Wilkinson for permission to cite unpublished results. The skilled technical assistance of Miss Mary Anderzon and Miss Berit Lindholm is gratefully acknowledged.

This work was supported by grants nos. B67-40X-656-02 and B67-40X-786-02 from the Swedish Medical Research Council and from Emil och Wera Cornells Stiftelse.

\section{REFERENCES}

Adams, M. H. (1959). Bacteriophages. New York: Interscience Publishers, Inc.

BeckmanN, I., LÜDERITZ, O. \& Westphal, O. (1964). Zur Immunchemie der somatischen Antigene von Enterobacteriaceae. IX. Serologische Typisierung von Salmonella-R-Antigenen. Biochem. $Z$. 339, 401 .

BRANDIS, H. (1966). Untersuchungen über die Wirkung von R-spezifischen Bakteriophagen auf R-Formen von S. paratyphi B. Zentbl. Bakt. ParasitKde. (Abt. I. Orig.) 199, 185.

Holme, T., Lindberg, A. A., GaregG, P. J. \& OnN, T. (1968). Chemical composition of cell-wall polysaccharide of rough mutants of Salmonella typhimurium. J. gen. Microbiol. 52, 45.

LüDERITZ, O., BECKMANN, I. \& WeSTPHAL, O. (I964). Zur Immunchemie der somatischen Antigene von Enterobacteriaceae. X. R-spezifische Strukturen in Salmonella-O-Antigenen. Biochem. $Z$. 339. $4 \mathrm{I} 6$.

Lüderitz, O., Galanos, C., Risse, H. J., Ruschmann, E., Schlecht, S., Schmidt, G., SchulteHolthausen, H., Wheat, R., Westphal, O. \& Schlosshardt, J. (I966a). Structural relationships of Salmonella O and R antigens. Ann. N.Y. Acad. Sci. 133, 349.

LüDeritz, O., STaub, A. M. \& WestPhal, O. (I966 b). Immunochemistry of $O$ and R antigens of Salmonella and related Enterobacteriaceae. Bact. Rev. 3o, 192.

Naide, Y., Nikaido, H., Mäkelä, P. H., Wilkinson, R. G. \& Stocker, B. A. D. (1965). Semirough strains of Salmonella. Proc. natn. Acad. Sci. U.S.A. 53, 147.

Nikaido, H., Nikaido, K., Subbaiah, T. V. \& Stocker, B. A. D. (I964). Rough mutants of Salmonella typhimurium. 3. Enzymatic synthesis of nucleotide sugar compounds. Nature, Lond. 201, I $30 \mathrm{I}$.

SHEDlovsky, A. \& BRENNER, S. (I963). A chemical basis for the host-induced modification of T-even bacteriophages. Proc. natn. Acad. Sci. U.S.A. 50, 300.

Stocker, B. A. D., Wilkinson, R. G. \& MäKelÄ, P. H. (1966). Genetic aspects of biosynthesis and structure of Salmonella somatic polysaccharide. Ann. N.Y. Acad. Sci. 133, 334.

Subbaiah, T. V. \& STOCKer, B. A. D. (1964). Rough mutants of Salmonella typhimurium. I. Genetics. Nature, Lond. 201, 298.

WestPhal, O., LüDeritz, O. \& Bister, F. (1952). Über die Extraktion von Bakterien mit Phenol/ Wasser. Z. Naturf. $7 b, \mathrm{I} 48$.

Wilkinson, R. G. \& Stocker, B. A. D. (1964). Phage patterns of rough mutants of Salmonella typhimurium strain LT2. J. gen. Microbiol. 37, i.

ZINDER, N. D. \& LeDERBERG, J. (1952). Genetic exchange in Salmonella. J. Bact. 64, 679. 